\title{
BIAS-REDUCED ESTIMATORS OF THE WEIBULL TAIL-COEFFICIENT
}

\author{
Jean Diebolt ${ }^{(1)}$, Laurent Gardes ${ }^{(2)}$, Stéphane Girard ${ }^{(3)}$ and Armelle \\ Guillou $^{(4)}$
}

(1) CNRS, Université de Marne-la-Vallée

Équipe d'Analyse et de Mathématiques Appliquées

5, boulevard Descartes, Batiment Copernic

Champs-sur-Marne

77454 Marne-la-Vallée Cedex 2

(2) Université Grenoble 2, LabSAD, 1251 Avenue centrale

B.P. 47, 38040 Grenoble Cedex 9

(3) Université Grenoble 1, LMC-IMAG, 51 rue des Mathématiques

B.P. 53, 38041 Grenoble Cedex 9

(4) Université Paris VI

Laboratoire de Statistique Théorique et Appliquée

Boîte 158

175 rue du Chevaleret

75013 Paris

\begin{abstract}
In this paper, we consider the problem of the estimation of a Weibull tail-coefficient $\theta$. In particular, we propose a regression model, from which we derive a bias-reduced estimator of $\theta$. This estimator is based on a least-squares approach. The asymptotic normality of this estimator is also established. A small simulation study is provided in order to prove its efficiency.
\end{abstract}

Key words and phrases. Weibull tail-coefficient, Bias-reduction, least-squares approach, asymptotic normality.

AMS Subject classifications. 62G05, 62G20, 62G30. 


\section{Introduction}

Let $X_{1}, \ldots, X_{n}$ be a sequence of independent and identically distributed random variables with distribution function $F$, and let $X_{1, n} \leq \ldots \leq X_{n, n}$ denote the order statistics associated to this sample.

In the present paper, we address the problem of estimating the Weibull tail-coefficient $\theta>0$ defined as

$$
1-F(x)=\exp (-H(x)) \text { with } H^{-1}(x):=\inf \{t: H(t) \geq x\}=x^{\theta} \ell(x),
$$

where $\ell$ is a slowly varying function at infinity satisfying

$$
\frac{\ell(\lambda x)}{\ell(x)} \longrightarrow 1 \text {, as } x \rightarrow \infty, \text { for all } \lambda>0 .
$$

[2] investigated this estimation problem and proposed the following estimator of $\theta$ :

$$
\widetilde{\theta}_{n}\left(k_{n}\right)=\frac{\sum_{i=1}^{k_{n}}\left(\log X_{n-i+1, n}-\log X_{n-k_{n}+1, n}\right)}{\sum_{i=1}^{k_{n}}\left(\log \log (n / i)-\log \log \left(n / k_{n}\right)\right)},
$$

where $k_{n}$ is an intermediate sequence, i.e. a sequence such that $k_{n} \rightarrow \infty$ and $k_{n} / n \rightarrow 0$ as $n \rightarrow \infty$.

We refer to [15, 13, 4] and [7] for other propositions and to [3] for Local Asymptotic Normality (LAN) results. Estimator (3) is closed in spirit to the Hill estimator [17] in the case of Pareto-type distributions. In [15] , the asymptotic normality of $\widetilde{\theta}_{n}\left(k_{n}\right)$ is established under suitable assumptions. To prove such a result, a second-order condition is required in order to specify the bias-term. This assumption can be expressed in terms of the slowly varying function $\ell$ as follows:

Assumption $\left(R_{\ell}(b, \rho)\right)$ There exists a constant $\rho<0$ and a rate function $b$ satisfying $b(x) \rightarrow 0$ as $x \rightarrow \infty$, such that for all $\varepsilon>0$ and $1<A<\infty$, we have

$$
\sup _{\lambda \in[1, A]}\left|\frac{\log (\ell(\lambda x) / \ell(x))}{b(x) K_{\rho}(\lambda)}-1\right| \leq \varepsilon, \quad \text { for } x \text { sufficiently large, }
$$

with $K_{\rho}(\lambda)=\int_{1}^{\lambda} t^{\rho-1} d t$ 
It can be shown that necessarily $|b|$ is regularly varying with index $\rho$ [14]. Moreover, we focus on the case where the convergence (2) is slow, and thus when the bias term in $\widetilde{\theta}_{n}\left(k_{n}\right)$ is large. This situation is described by the following assumption:

$$
x|b(x)| \rightarrow \infty \text { as } x \rightarrow \infty .
$$

Let us note that this condition implies $\rho \geq-1$. Gamma and Gaussian distributions fulfill (4), whereas Weibull distributions do not (see Table 1) since, in this case, the bias term vanishes.

Using this framework, we will establish rigorously in Section 2] the following approximation for the log-spacings of upper order statistics:

$$
\begin{aligned}
Z_{j} & :=j \log (n / j)\left(\log X_{n-j+1, n}-\log X_{n-j, n}\right) \\
& \approx\left(\theta+b\left(\log \left(n / k_{n}\right)\right)\left(\frac{\log (n / j)}{\log \left(n / k_{n}\right)}\right)^{\rho}\right) f_{j},
\end{aligned}
$$

for $1 \leq j \leq k_{n}$, where $\left(f_{1}, \ldots, f_{k_{n}}\right)$ is a vector of independent and standard exponentially distributed random variables. This exponential regression model is similar to the ones proposed by [5, 6] and [11] in the case of Pareto-type distributions. Ignoring $b\left(\log \left(n / k_{n}\right)\right)\left(\frac{\log (n / j)}{\log \left(n / k_{n}\right)}\right)^{\rho}$ in (5) leads to the maximum likelihood estimator

$$
\breve{\theta}_{n}\left(k_{n}\right)=\frac{1}{k_{n}} \sum_{j=1}^{k_{n}} Z_{j}
$$

which turns out to be an alternative estimator of $\widetilde{\theta}_{n}\left(k_{n}\right)$. The full model (5) allows us to generate bias-corrected estimates $\widehat{\theta}_{n}\left(k_{n}\right)$ for $\theta$ through maximum likelihood estimation of $\theta, b\left(\log n / k_{n}\right)$ and $\rho$ for each $1 \leq k_{n} \leq n-1$. An alternative to this approach consists in using a canonical choice for $\rho$ and to estimate the two other parameters by a least-squares method (LS). For the canonical choice of $\rho$, we can use for instance the value -1 , which is the same as the one proposed by [11] for the regression model in the case of Pareto-type distributions. The asymptotic normality of the resulting LS-estimator is established in Section 3, An adaptive selection method for $k_{n}$ in $\check{\theta}_{n}\left(k_{n}\right)$ is also 
derived. In order to illustrate the usefulness of these results, we provide a simulation study in Section 4 as well as an application to a real data set in Section 5. The proofs of our results are postponed to Section 7

\section{Exponential regression model}

In this section, we formalize (5). First, remark that

$$
F^{-1}(x)=[-\log (1-x)]^{\theta} \ell(-\log (1-x)) .
$$

Since $X_{n-j+1, n} \stackrel{d}{=} F^{-1}\left(U_{n-j+1, n}\right), 1 \leq j \leq k_{n}$, where $U_{j, n}$ denotes the $j$-th order statistic of a uniform sample of size $n$, we have

$$
X_{n-j+1, n} \stackrel{d}{=}\left[-\log \left(1-U_{n-j+1, n}\right)\right]^{\theta} \ell\left(-\log \left(1-U_{n-j+1, n}\right)\right)
$$

which implies that

$$
\log X_{n-j+1, n} \stackrel{d}{=} \theta \log \left[-\log \left(1-U_{n-j+1, n}\right)\right]+\log \left[\ell\left(-\log \left(1-U_{n-j+1, n}\right)\right)\right] .
$$

Moreover, considering the order statistics from an independent standard exponential sample, $E_{n-j+1, n} \stackrel{d}{=}-\log \left(1-U_{n-j+1, n}\right)$. Therefore

$$
\begin{aligned}
\log X_{n-j+1, n} & \stackrel{d}{=} \theta \log \left(E_{n-j+1, n}\right)+\log \left[\ell\left(E_{n-j+1, n}\right)\right] \\
& =: A_{n}(j)+B_{n}(j) .
\end{aligned}
$$

Recall that $Z_{j}=j \log (n / j)\left(\log X_{n-j+1, n}-\log X_{n-j, n}\right), 1 \leq j \leq k_{n}$. Then, our basic result now reads as follows.

Theorem 1 Suppose (1) holds together with $\left(R_{\ell}(b, \rho)\right)$ and (4). Then, if $k_{n} \rightarrow \infty$ and $\log k_{n} / \log n \rightarrow 0$, we have

$$
\sup _{1 \leq j \leq k_{n}}\left|Z_{j}-\left(\theta+b\left(\log \left(n / k_{n}\right)\right)\left(\frac{\log (n / j)}{\log \left(n / k_{n}\right)}\right)^{\rho}\right) f_{j}\right|=o_{\mathbb{P}}\left(b\left(\log \left(n / k_{n}\right)\right)\right),
$$

where $\left(f_{1}, \ldots, f_{k_{n}}\right)$ is a vector of independent and standard exponentially distributed random variables.

The proof of this theorem is based on the following two lemmas: 
Lemma 1 Suppose (1) holds together with $\left(R_{\ell}(b, \rho)\right)$ and (4). Then, if $k_{n} \rightarrow \infty$ and $k_{n} / n \rightarrow 0$, we have

$$
\sup _{1 \leq j \leq k_{n}}\left|j \log (n / j)\left[A_{n}(j)-A_{n}(j+1)\right]-\theta f_{j}\right|=o_{\mathbb{P}}\left(b\left(\log \left(n / k_{n}\right)\right)\right),
$$

and

Lemma 2 Suppose (1) holds together with $\left(R_{\ell}(b, \rho)\right)$. Then, if $k_{n} \rightarrow \infty$ and $\log k_{n} / \log n \rightarrow 0$, we have

$$
\begin{aligned}
& \sup _{1 \leq j \leq k_{n}}\left|j \log (n / j)\left[B_{n}(j)-B_{n}(j+1)\right]-b\left(\log \left(n / k_{n}\right)\right)\left(\frac{\log (n / j)}{\log \left(n / k_{n}\right)}\right)^{\rho} f_{j}\right| \\
& =o_{\mathbb{P}}\left(b\left(\log \left(n / k_{n}\right)\right)\right) .
\end{aligned}
$$

The proof of these lemmas is postponed to Section 7

Corollary 1 Under the assumptions of Theorem 1, we also have

$$
\sup _{1 \leq j \leq k_{n}}\left|Z_{j}-\left(\theta+b\left(\log \left(n / k_{n}\right)\right)\left(\frac{\log (n / j)}{\log \left(n / k_{n}\right)}\right)^{-1}\right) f_{j}\right|=o_{\mathbb{P}}\left(b\left(\log \left(n / k_{n}\right)\right)\right) \text {, }
$$

where $\left(f_{1}, \ldots, f_{k_{n}}\right)$ is a vector of independent and standard exponentially distributed random variables.

This implies that one can plug the canonical choice $\rho=-1$ in the regression model (6) without perturbing the approximation. From model (6) we can easily deduce the asymptotic normality of the estimator $\breve{\theta}_{n}\left(k_{n}\right)$, given in the next theorem:

Theorem 2 Suppose (1) holds together with $\left(R_{\ell}(b, \rho)\right)$ and (4). Then, if $k_{n} \rightarrow \infty, \sqrt{k_{n}} b\left(\log \left(n / k_{n}\right)\right) \rightarrow \lambda \in \mathbb{R}$ and, if $\lambda=0, \log k_{n} / \log n \rightarrow 0$, we have

$$
\sqrt{k_{n}}\left(\check{\theta}_{n}\left(k_{n}\right)-\theta-b\left(\log \left(n / k_{n}\right)\right) \frac{1}{k_{n}} \sum_{j=1}^{k_{n}}\left(\frac{\log (n / j)}{\log \left(n / k_{n}\right)}\right)^{\rho}\right) \stackrel{d}{\longrightarrow} \mathcal{N}\left(0, \theta^{2}\right) .
$$

The Asymptotic Mean Squared Error (AMSE) associated to $\check{\theta}_{n}\left(k_{n}\right)$ is thus given by:

$$
\operatorname{AMSE}\left(\check{\theta}_{n}\left(k_{n}\right)\right)=\frac{\theta^{2}}{k_{n}}+\left(b\left(\log \left(n / k_{n}\right)\right) \frac{1}{k_{n}} \sum_{j=1}^{k_{n}}\left(\frac{\log (n / j)}{\log \left(n / k_{n}\right)}\right)^{\rho}\right)^{2} .
$$


This model (6) now plays the central role in the remainder of this paper. First, it allows us to generate bias-corrected estimates of $\theta$. Second, it leads to the number of upper order statistics $k_{n}$ to be used in $\check{\theta}_{n}\left(k_{n}\right)$ by minimizing the AMSE given by (7) after replacing $\theta$, $b$ and $\rho$ by estimators. These two points are described in the next section.

\section{Bias-reduced estimates of $\theta$ and adaptive selection of $k_{n}$}

In order to reduce the bias of the estimator $\breve{\theta}_{n}\left(k_{n}\right)$, we can either estimate simultaneously $\theta, b\left(\log n / k_{n}\right)$ and $\rho$ by a maximum likelihood method or estimate $\theta$ and $b$ by a least-squares approach after substituting a canonical choice for $\rho$. In fact, this second-order parameter is difficult to estimate in practice and we can easily check by simulations that fixing its value does not much influence the result. This problem has already been discussed in [5, 6] and [11] where similar observations have been made in the case of Pareto-type distributions. The canonical choice $\rho=-1$ is often used although other choices could be motivated performing a model selection.

In all the sequel, we will estimate $\theta$ and $b\left(\log \left(n / k_{n}\right)\right)$ by a LSmethod after substituting $\rho$ with the value -1 . In that case, we find the following LS-estimators:

$$
\left\{\begin{array}{l}
\widehat{\theta}_{n}\left(k_{n}\right)=\bar{Z}_{k_{n}}-\widehat{b}\left(\log \left(n / k_{n}\right)\right) \bar{x}_{k_{n}} \\
\widehat{b}\left(\log \left(n / k_{n}\right)\right)=\frac{\sum_{j=1}^{k_{n}}\left(x_{j}-\bar{x}_{k_{n}}\right) Z_{j}}{\sum_{j=1}^{k_{n}}\left(x_{j}-\bar{x}_{k_{n}}\right)^{2}}
\end{array}\right.
$$

where $x_{j}=\left(\frac{\log (n / j)}{\log \left(n / k_{n}\right)}\right)^{-1}, \bar{x}_{k_{n}}=\frac{1}{k_{n}} \sum_{j=1}^{k_{n}} x_{j}$ and $\bar{Z}_{k_{n}}=\frac{1}{k_{n}} \sum_{j=1}^{k_{n}} Z_{j}$. Our next goal is to establish, under suitable assumptions, the asymptotic normality of $\widehat{\theta}_{n}\left(k_{n}\right)$. This is done in the following theorem.

Theorem 3 Suppose (1) holds together with $\left(R_{\ell}(b, \rho)\right)$ and (4). Then, if 
$k_{n} \rightarrow \infty$ such that

$$
\begin{aligned}
& \frac{\sqrt{k_{n}}}{\log \left(n / k_{n}\right)} b\left(\log \left(n / k_{n}\right)\right) \rightarrow \Lambda \in \mathbb{R} \text { and, if } \Lambda=0, \\
& \frac{\log ^{2} k_{n}}{\log \left(n / k_{n}\right)} \rightarrow 0 \text { and } \frac{\sqrt{k_{n}}}{\log \left(n / k_{n}\right)} \rightarrow \infty,
\end{aligned}
$$

we have

$$
\frac{\sqrt{k_{n}}}{\log \left(n / k_{n}\right)}\left(\widehat{\theta}_{n}\left(k_{n}\right)-\theta\right) \stackrel{d}{\longrightarrow} \mathcal{N}\left(0, \theta^{2}\right) .
$$

Remark that the rate of convergence of $\check{\theta}_{n}\left(k_{n}\right)$ is the same as the one of $\widehat{\theta}_{n}\left(k_{n}\right)$ in the cases where both $\lambda$ and $\Lambda$ are not equal to 0 . The proof of this theorem is postponed to Section 7 .

We can also take benefit of the estimation of $b\left(\log n / k_{n}\right)$ by estimating the AMSE given in (7) by:

$$
\widehat{A M S E}\left(\check{\theta}_{n}\left(k_{n}\right)\right)=\frac{\left(\widehat{\theta}_{n}\left(k_{n}\right)\right)^{2}}{k_{n}}+\left(\widehat{b}\left(\log \left(n / k_{n}\right)\right) \frac{1}{k_{n}} \sum_{j=1}^{k_{n}}\left(\frac{\log (n / j)}{\log \left(n / k_{n}\right)}\right)^{-1}\right)^{2} .
$$

Then, the intermediate sequence $k_{n}$ can be selected by minimizing the previous quantity:

$$
\hat{k}_{n}=\arg \min _{k_{n}} \widehat{A M S} E\left(\check{\theta}_{n}\left(k_{n}\right)\right) .
$$

This adaptive procedure for selecting the number of upper order statistics is in the same spirit as the one used by [19] in the context of the extreme value index estimation.

In order to illustrate the usefulness of the bias reduction and of the selection procedure, we provide a simulation study in the next section.

\section{A simulation study}

First, the finite sample performances of the estimators $\widehat{\theta}_{n}\left(k_{n}\right), \widetilde{\theta}_{n}\left(k_{n}\right)$ and $\check{\theta}_{n}\left(k_{n}\right)$ are investigated on 6 different distributions: $\Gamma(0.25,1)$, 
$\Gamma(4,1),|\mathcal{N}|(0,1), \mathcal{W}(0.25,0.25), \mathcal{W}(4,4)$ and $\mathcal{D}(1,0.5)$, see the appendix for the definition of the latter distribution. We limit ourselves to these three estimators, since it is shown in [15] that $\widetilde{\theta}_{n}\left(k_{n}\right)$ gives better results than the other approaches [4, 7]. In each case, $N=100$ samples $\left(\mathcal{X}_{n, i}\right)_{i=1, \ldots, N}$ of size $n=500$ were simulated. On each sample $\left(\mathcal{X}_{n, i}\right)$, the estimates $\widehat{\theta}_{n, i}\left(k_{n}\right), \widetilde{\theta}_{n, i}\left(k_{n}\right)$ and $\breve{\theta}_{n, i}\left(k_{n}\right)$ were computed for $k_{n}=2, \ldots, 360$. Finally, the Hill-type plots were built by drawing the points

$$
\left(k_{n}, \frac{1}{N} \sum_{i=1}^{N} \widehat{\theta}_{n, i}\left(k_{n}\right)\right),\left(k_{n}, \frac{1}{N} \sum_{i=1}^{N} \widetilde{\theta}_{n, i}\left(k_{n}\right)\right) \text { and }\left(k_{n}, \frac{1}{N} \sum_{i=1}^{N} \check{\theta}_{n, i}\left(k_{n}\right)\right) \text {. }
$$

We also present the associated MSE plots obtained by plotting the points

$$
\begin{aligned}
& \left(k_{n}, \frac{1}{N} \sum_{i=1}^{N}\left(\widehat{\theta}_{n, i}\left(k_{n}\right)-\theta\right)^{2}\right),\left(k_{n}, \frac{1}{N} \sum_{i=1}^{N}\left(\widetilde{\theta}_{n, i}\left(k_{n}\right)-\theta\right)^{2}\right) \text { and } \\
& \left(k_{n}, \frac{1}{N} \sum_{i=1}^{N}\left(\check{\theta}_{n, i}\left(k_{n}\right)-\theta\right)^{2}\right) .
\end{aligned}
$$

The results are presented on figures 1-6. In all the plots, the graphs associated to $\widetilde{\theta}_{n}\left(k_{n}\right)$ and $\breve{\theta}_{n}\left(k_{n}\right)$ are similar, with a slightly better behavior of $\check{\theta}_{n}\left(k_{n}\right)$. The bias corrected estimator $\widehat{\theta}_{n}\left(k_{n}\right)$ always yields a smaller bias than the two previous ones leading to better results for Gamma, Gaussian and $\mathcal{D}$ distributions (figures 1-4), even though a wrong value of $\rho$ is used (figure 4). On Weibull distributions, where the bias function is zero, (figures [5-6), it presents a larger variance. Second, we investigate the behavior of the adaptive procedure for selecting the number of upper order statistics in $\check{\theta}_{n}\left(k_{n}\right)$. For $i=$ $1, \ldots, N$, we denote by

$$
\widehat{k_{n, i}}=\arg \min _{k_{n} \in[1,350]} \widehat{A M S} E\left(\check{\theta}_{n, i}\left(k_{n}\right)\right)
$$

the value selected on the sample $\left(\mathcal{X}_{n, i}\right)$. Note that, as in [19], in our simulations, we limited the range from which $k_{n}$ is selected to $\{1, \ldots, 350\}$. The mean and the standard deviation of this estimation 
on the $N$ samples are given by

$$
\mu\left(\widehat{k_{n}}\right)=\frac{1}{N} \sum_{i=1}^{N} \widehat{k_{n, i}} \text { and } \sigma\left(\widehat{k_{n}}\right)=\sqrt{\frac{1}{N} \sum_{i=1}^{N}\left(\widehat{k_{n, i}}-\mu\left(\widehat{k_{n}}\right)\right)^{2}} .
$$

As a comparison, we introduce the value that would be obtained by minimizing the true AMSE:

$$
k_{n}^{\text {opt }}=\arg \min _{k_{n} \in[1,350]} \operatorname{AMSE}\left(\check{\theta}_{n}\left(k_{n}\right)\right) .
$$

On each sample $\left(X_{n, i}\right)$, the estimation of $\theta$ obtained with the selected parameter $\widehat{k_{n, i}}$ is given by $\breve{\theta}_{n, i}\left(\widehat{k_{n, i}}\right)$. The associated empirical mean and standard deviation are:

$$
\mu\left(\check{\theta}_{n}\right)=\frac{1}{N} \sum_{i=1}^{N} \check{\theta}_{n, i}\left(\widehat{k_{n, i}}\right) \text { and } \sigma\left(\check{\theta}_{n}\right)=\sqrt{\frac{1}{N} \sum_{i=1}^{N}\left(\check{\theta}_{n, i}\left(\widehat{k_{n, i}}\right)-\mu\left(\check{\theta}_{n}\right)\right)^{2}} .
$$

Finally, to assess the quality of the selection procedure, we compute the ratio $R_{n}$ of the empirical root mean squared error of $\check{\theta}_{n}\left(\widehat{k}_{n}\right)$ and the minimal empirical root mean squared error of $\check{\theta}_{n}\left(k_{n}\right)$ :

$$
R_{n}^{2}=\sum_{i=1}^{N}\left(\check{\theta}_{n, i}\left(\widehat{k_{n, i}}\right)-\theta\right)^{2} \mid \min _{k_{n} \in[1,350]} \sum_{i=1}^{N}\left(\check{\theta}_{n, i}\left(k_{n}\right)-\theta\right)^{2} .
$$

Results are presented in Table 2. It appears that $R_{n}$ is usually "close" to 1 , except for Weibull distributions. In this case, large values of $R_{n}$ together with large values of $\mu\left(\hat{k}_{n}\right)$ indicate that the optimal $k_{n}$ is larger than 350 observations.

\section{Real data}

Here, the good performance of the adaptive selection procedure is illustrated through the analysis of extreme events on a benchmark real data set. Nidd river data are widely used in extreme value studies [18, 8]. The raw data consist in 154 exceedances of the level $65 \mathrm{~m}^{3} \mathrm{~s}^{-1}$ by the river Nidd (Yorkshire, England) during the period 1934-1969 
(35 years). The $N$-year return level is the water level which is exceeded on average once in $N$ years. Hydrologists need to estimate extreme quantiles in order to predict return levels over long periods. According to [18], the Nidd data may reasonably be assumed to come from a distribution in the Gumbel maximum domain of attraction. This suggests to consider Weibull tail-distributions as a possible model for such data. The adaptive selection procedure yields $\widehat{k}_{n}=29$. The resulting quantile-quantile plot (obtained by plotting the points $\left(\log \log (n / i), \log \left(X_{n-i+1, n}\right)\right)$ for $\left.i=1, \ldots, \widehat{k}_{n}-1\right)$ is approximatively linear (see Figure Z), indicating a good fit of the Weibull tail-distribution for $x \geq X_{n-\hat{k}_{n}+1, n}$. We obtained $\check{\theta}_{n}\left(\hat{k}_{n}\right) \simeq \widetilde{\theta}_{n}\left(\hat{k}_{n}\right) \simeq 0.89$. One can plug this result in the Weissman-type extreme quantile estimator proposed in [12] to obtain $366 \mathrm{~m}^{3} \mathrm{~s}^{-1}$ as an estimation of the 100-year return level. Note that this result is in accordance with the results obtained by profile-likelihood or Bayesian methods, see [9] or [8].

\section{Concluding remarks}

In this paper, we introduce a regression model, from which we derive a bias-reduced estimator for the Weibull tail-coefficient $\theta$. Its asymptotic normality is established and an adaptive selection procedure for $k_{n}$ is proposed. The efficiency of our approach is illustrated in a simulation study and on a real data set. However, in many cases of practical interest, the problem of estimating a quantile $x_{p_{n}}=F^{-1}\left(1-p_{n}\right)$, with $p_{n}<1 / n$, is much more important. Such a problem has already been studied in [12] where the following Weissman-type estimator has been introduced

$$
\tilde{x}_{p_{n}}\left(k_{n}\right)=X_{n-k_{n}+1, n}\left(\frac{\log \left(1 / p_{n}\right)}{\log \left(n / k_{n}\right)}\right)^{\widetilde{\theta}_{n}\left(k_{n}\right)} .
$$

It is, however, desirable to refine $\widetilde{x}_{p_{n}}\left(k_{n}\right)$ with the additional information about the slowly varying function $\ell$ that is provided by the LS-estimates for $\theta$ and $b$. To this aim, condition $\left(R_{\ell}(b, \rho)\right)$ is used to approximate the ratio $F^{-1}\left(1-p_{n}\right) / X_{n-k_{n}+1, n}$, noting that

$$
X_{n-k_{n}+1, n} \stackrel{d}{=} F^{-1}\left(U_{n-k_{n}+1, n}\right),
$$


with $U_{1, n} \leq \ldots \leq U_{n, n}$ the order statistics of a uniform $(0,1)$ sample of size $n$,

$$
\begin{aligned}
& \frac{x_{p_{n}}}{X_{n-k_{n}+1, n}} \stackrel{d}{=} \frac{F^{-1}\left(1-p_{n}\right)}{F^{-1}\left(U_{n-k_{n}+1, n}\right)} \\
& \stackrel{d}{=} \frac{\left(-\log p_{n}\right)^{\theta}}{\left(-\log \left(1-U_{n-k_{n}+1, n}\right)\right)^{\theta}} \frac{\ell\left(-\log p_{n}\right)}{\ell\left(-\log \left(1-U_{n-k_{n}+1, n}\right)\right)} \\
& \stackrel{d}{\simeq}\left(\frac{\log \left(1 / p_{n}\right)}{\log \left(n / k_{n}\right)}\right)^{\theta} \exp \left[b\left(\log \left(n / k_{n}\right)\right) \frac{\left(\frac{\log \left(1 / p_{n}\right)}{\log \left(n / k_{n}\right)}\right)^{\rho}-1}{\rho}\right] \text {. }
\end{aligned}
$$

The last step follows from replacing $U_{k_{n}+1, n}$ (resp. $E_{n-k_{n}+1, n}$ ) by $k_{n} / n$ (resp. $\left.\log \left(n / k_{n}\right)\right)$. Hence, we arrive at the following estimator for extreme quantiles

$$
\widehat{x}_{p_{n}}\left(k_{n}\right)=X_{n-k_{n}+1, n}\left(\frac{\log \left(1 / p_{n}\right)}{\log \left(n / k_{n}\right)}\right)^{\widehat{\theta}_{n}\left(k_{n}\right)} \exp \left[\widehat{b}\left(\log \left(n / k_{n}\right)\right) \frac{\left(\frac{\log \left(1 / p_{n}\right)}{\log \left(n / k_{n}\right)}\right)^{\widehat{\rho}}-1}{\widehat{\rho}}\right],
$$

which is similar to the estimator proposed by [20] in the case of Pareto-type distributions. Here, the LS-estimators of $\theta$ and $b$ can be used after substituting $\rho$ by the canonical choice -1 . The study of the asymptotic properties of such an estimator is the aim of [10].

\section{$7 \quad$ Proofs of our results}

\subsection{Preliminary lemmas}

Lemma 3 For all $1 \leq j \leq k_{n}$ such that $k_{n} \rightarrow \infty$ and $k_{n} / n \rightarrow 0$, we have

$$
\frac{E_{n-j, n}}{\log (n / j)}=1+O_{\mathbb{P}}\left(\frac{1}{\log \left(n / k_{n}\right)}\right) \text { uniformly in } j \text {. }
$$

Proof of Lemma 3, According to Rényi's representation, we have

$$
E_{n-j, n} \stackrel{d}{=} \sum_{\ell=1}^{n-j+1} \frac{f_{n-\ell-j+1}}{\ell+j-1}
$$


where $f_{j} \stackrel{\text { i.i.d. }}{\sim} \operatorname{Exp}(1)$. Since

$$
\operatorname{Var}\left(\sum_{\ell=1}^{n-j+1} \frac{f_{n-\ell-j+1}}{\ell+j-1}\right)=O(1)
$$

denoting

$$
T_{j, n}:=\sum_{\ell=1}^{n-j+1}\left[\frac{f_{n-\ell-j+1}}{\ell+j-1}-\mathbb{E} \frac{f_{n-\ell-j+1}}{\ell+j-1}\right],
$$

we have, using Kolmogorov's inequality [21] (p.183), that

$$
\mathbb{P}\left(\max _{1 \leq j \leq k_{n}}\left|T_{j, n}\right| \geq \lambda\right) \leq \frac{\operatorname{Var}\left(T_{1, n}\right)}{\lambda^{2}}, \quad \lambda>0 .
$$

This implies that $T_{j, n}=O_{\mathbb{P}}(1)$ uniformly in $j$. Taking into account the fact that

$$
\left|\sum_{\ell=j}^{n} \frac{1}{\ell}-\log (n / j)\right|=O(1) \quad \text { uniformly in } j, 1 \leq j \leq k_{n},
$$

it is easy to deduce Lemma 3 ,

Let us introduce the $E_{m}$-function defined by the integral

$$
E_{m}(x):=\int_{1}^{\infty} \frac{e^{-x t}}{t^{m}} d t
$$

for a positive integer $m$. The asymptotic expansion of this integral is given in the following lemma.

Lemma 4 As $x \rightarrow \infty$, for any fixed positive integers $m$ and $p$, we have

$$
E_{m}(x)=\frac{e^{-x}}{x}\left\{1-\frac{m}{x}+\ldots+(-1)^{p} \frac{m(m+1) \ldots(m+p-1)}{x^{p}}+O\left(\frac{1}{x^{p+1}}\right)\right\} .
$$

The proof of this lemma is straightforward from [1] p. 227-233 and the $\mathrm{O}$-term can be obtained by a Taylor expansion with an integral remainder. Denote

$$
\mu_{p}:=\frac{1}{k_{n}} \sum_{j=1}^{k_{n}}\left(x_{j}-\bar{x}_{k_{n}}\right)^{p}, p \in \mathbb{N}^{*} .
$$

The next lemma provides a first order expansion of this Riemman sum. 
Lemma 5 If $k_{n} \rightarrow \infty, k_{n} / n \rightarrow 0, \frac{k_{n}}{\log \left(n / k_{n}\right)} \rightarrow \infty$ and $\frac{\log ^{2} k_{n}}{\log \left(n / k_{n}\right)} \rightarrow 0$, then

$$
\mu_{p} \sim C_{p}\left(\log \left(n / k_{n}\right)\right)^{-p} \text { as } n \rightarrow \infty \text {, where } C_{p}=\int_{0}^{1}(\log x+1)^{p} d x<\infty .
$$

Proof of Lemma 5, Denote $\alpha_{n}=\frac{1}{\log \left(n / k_{n}\right)}$. Then $\bar{x}_{k_{n}}$ can be rewritten as

$$
\bar{x}_{k_{n}}=\frac{1}{k_{n}}+\left(\frac{1}{k_{n}} \sum_{j=1}^{k_{n}-1} f_{n}\left(j / k_{n}\right)-\int_{0}^{1} f_{n}(x) d x\right)+\int_{0}^{1} f_{n}(x) d x=: \frac{1}{k_{n}}+T_{1}+T_{2},
$$

where $f_{n}(x)=\left(1-\alpha_{n} \log x\right)^{-1}, x \in[0,1]$. Denoting by $f_{n}^{(i)}, i \in\{1,2\}$, the $i$ th derivative of $f_{n}$, we infer that

$$
\begin{aligned}
T_{1} & =\sum_{j=1}^{k_{n}-1} \int_{j / k_{n}}^{(j+1) / k_{n}}\left(j / k_{n}-t\right) f_{n}^{(1)}\left(j / k_{n}\right) d t \\
& +\sum_{j=1}^{k_{n}-1} \int_{j / k_{n}}^{(j+1) / k_{n}} \int_{j / k_{n}}^{t}(x-t) f_{n}^{(2)}(x) d x d t+\int_{0}^{1 / k_{n}} f_{n}(x) d x \\
& =: T_{3}+T_{4}+T_{5} .
\end{aligned}
$$

Remark that

$$
\begin{aligned}
T_{3} & =-\frac{1}{2 k_{n}}\left(\frac{1}{k_{n}} \sum_{j=1}^{k_{n}-1} f_{n}^{(1)}\left(j / k_{n}\right)-\int_{1 / k_{n}}^{1} f_{n}^{(1)}(t) d t\right)-\frac{1}{2 k_{n}} \int_{1 / k_{n}}^{1} f_{n}^{(1)}(t) d t \\
& =:-\frac{1}{2 k_{n}} T_{6}+T_{7} .
\end{aligned}
$$

Since $f_{n}^{(1)}$ is positive and decreasing on $\left[\frac{1}{k_{n}}, 1\right]$ for $n$ sufficiently large, we can prove that

$$
\begin{aligned}
\left|T_{4}\right| & \leq \frac{1}{2 k_{n}^{2}}\left|f_{n}^{(1)}\left(1 / k_{n}\right)-f_{n}^{(1)}(1)\right|=o\left(1 / k_{n}\right), \\
T_{5} & =O\left(1 / k_{n}\right), \\
\left|T_{6}\right| & \leq \frac{1}{k_{n}}\left|f_{n}^{(1)}\left(1 / k_{n}\right)-f_{n}^{(1)}(1)\right|=o(1), \\
T_{7} & =-\frac{1}{2 k_{n}}\left(f_{n}(1)-f_{n}\left(1 / k_{n}\right)\right)=o\left(1 / k_{n}\right),
\end{aligned}
$$


and consequently $T_{1}=O\left(1 / k_{n}\right)$. Besides, a direct application of Lemma 4 provides

$$
T_{2}=1-\alpha_{n}+O\left(\alpha_{n}^{2}\right) \text {. }
$$

Therefore $\bar{x}_{k_{n}}=1-\alpha_{n}+O\left(1 / k_{n}\right)+O\left(\alpha_{n}^{2}\right)$. Now, we can check that

$$
\left.\mu_{p}=\alpha_{n}^{p}\left\{\frac{1}{k_{n}} \sum_{j=1}^{k_{n}}\left(\log \left(j / k_{n}\right)\right)+1\right)^{p}+R_{n}\right\}
$$

where

$$
R_{n}=\frac{1}{k_{n}} \sum_{j=1}^{k_{n}-1}\left\{\left(\log \left(j / k_{n}\right)+1+\varepsilon_{n}\right)^{p}-\left(\log \left(j / k_{n}\right)+1\right)^{p}\right\}
$$

with $\varepsilon_{n}=O\left(\alpha_{n} \log ^{2} k_{n}\right)+O\left(\frac{1}{k \alpha_{n}}\right)$ which tends to 0 by assumption. Since $\frac{1}{C_{p}} \frac{1}{k_{n}} \sum_{j=1}^{k_{n}}\left(\log \left(j / k_{n}\right)+1\right)^{p} \rightarrow 1$, in order to conclude the proof of Lemma 5, we only have to remark that $R_{n} \rightarrow 0$.

\subsection{Proof of Lemma1}

Remark that

$$
\begin{aligned}
\alpha_{j, n} & :=j \log (n / j)\left[A_{n}(j)-A_{n}(j+1)\right] \\
& =\theta j \log (n / j) \log \left(E_{n-j+1, n} / E_{n-j, n}\right) \\
& =\theta \log (n / j) j\left(E_{n-j+1, n}-E_{n-j, n}\right) / E_{n-j, n}^{*} \\
& \stackrel{d}{=} \theta f_{j} \log (n / j) / E_{n-j, n}^{*}
\end{aligned}
$$

where $E_{n-j, n}^{*} \in\left[E_{n-j, n} ; E_{n-j+1, n}\right]$. Consequently, from Lemma 3 ,

$$
\begin{aligned}
\alpha_{j, n} & =\theta f_{j}+O_{\mathbb{P}}\left(\frac{1}{\log \left(n / k_{n}\right)}\right) \\
& =\theta f_{j}+o_{\mathbb{P}}\left(b\left(\log \left(n / k_{n}\right)\right)\right),
\end{aligned}
$$

by the assumption $x|b(x)| \rightarrow \infty$ as $x \rightarrow \infty$ with a $o_{\mathbb{P}}$-term which is uniform in $j$. Lemma 1 is therefore proved. 


\subsection{Proof of Lemma2}

We consider

$$
\beta_{j, n}:=j \log (n / j)\left[B_{n}(j)-B_{n}(j+1)\right] .
$$

In order to study this term, we will use the notations $\lambda_{1 j}=E_{n-j+1, n} / E_{n-k_{n}+1, n}$, $\lambda_{2 j}=E_{n-j, n} / E_{n-k_{n}+1, n}$ and $y_{k_{n}}=E_{n-k_{n}+1, n}$, and we rewrite $\beta_{j, n}$ as

$$
\beta_{j, n}=j \log (n / j)\left\{\log \ell\left(\lambda_{2 j} \frac{\lambda_{1 j}}{\lambda_{2 j}} y_{k_{n}}\right)-\log \ell\left(\lambda_{2 j} y_{k_{n}}\right)\right\} \text {. }
$$

It is clear that $1 \leq \lambda_{1 j} / \lambda_{2 j} \stackrel{\mathbb{P}}{\longrightarrow} 1$ uniformly in $j$ by Lemma 3 and therefore for $n \geq N_{0}, \lambda_{1 j} / \lambda_{2 j} \in[1,2]$ in probability. Under our assumption $\left(R_{\ell}(b, \rho)\right)$ on the slowly varying function, we deduce that

$$
\beta_{j, n}=j \log (n / j)\left\{b\left(\lambda_{2 j} y_{k_{n}}\right) K_{\rho}\left(\lambda_{1 j} / \lambda_{2 j}\right)\left(1+o_{\mathbb{P}}(1)\right)\right\} .
$$

Now, since $\lambda_{2 j} \stackrel{\mathbb{P}}{\longrightarrow} 1$ uniformly in $j$ and $b($.$) is regularly varying with$ index $\rho, b\left(\lambda_{2 j} y_{k_{n}}\right)=\lambda_{2 j}^{\rho} b\left(y_{k_{n}}\right)\left(1+o_{\mathbb{P}}(1)\right)$ with a $o_{\mathbb{P}}(1)$-term uniform in $j$. Therefore

$$
\beta_{j, n}=j \log (n / j) b\left(y_{k_{n}}\right)\left\{\lambda_{2 j}^{\rho} K_{\rho}\left(\lambda_{1 j} / \lambda_{2 j}\right)\left(1+o_{\mathbb{P}}(1)\right)\right\} .
$$

Again, uniformly in $j$,

$$
K_{\rho}\left(\lambda_{1 j} / \lambda_{2 j}\right)=\left(\lambda_{1 j} / \lambda_{2 j}-1\right)\left(1+o_{\mathbb{P}}(1)\right),
$$

which implies that $\beta_{j, n}$ can be rewritten as follows:

$$
\beta_{j, n}=-j \log (n / j) b\left(y_{k_{n}}\right)\left(\lambda_{2 j}-\lambda_{1 j}\right) \lambda_{2 j}^{\rho-1}\left(1+o_{\mathbb{P}}(1)\right) .
$$

Therefore, we have

$$
\beta_{j, n}=f_{j}\left(\frac{\log (n / j)}{\log \left(n / k_{n}\right)}\right)^{\rho} b\left(y_{k_{n}}\right)\left(1+o_{\mathbb{P}}(1)\right),
$$

with a $o_{\mathbb{P}}(1)$-term which is uniform in $j$. This achieves the proof of Lemma2.

Remark that, since $\frac{\log (n / j)}{\log \left(n / k_{n}\right)} \rightarrow 1$ uniformly in $j$, one also has

$$
\beta_{j, n}=f_{j}\left(\frac{\log (n / j)}{\log \left(n / k_{n}\right)}\right)^{-1} b\left(y_{k_{n}}\right)\left(1+o_{\mathbb{P}}(1)\right),
$$

with a $o_{\mathbb{P}}(1)$-term which is uniform in $j$, and this proves Corollary 1 , 


\subsection{Proof of Theorem 2}

From model (6), we infer that

$$
\begin{aligned}
& \sqrt{k_{n}}\left(\check{\theta}_{n}\left(k_{n}\right)-\theta-b\left(\log \left(n / k_{n}\right)\right) \frac{1}{k_{n}} \sum_{j=1}^{k_{n}}\left(\frac{\log (n / j)}{\log \left(n / k_{n}\right)}\right)^{\rho}\right) \\
& =\sqrt{k_{n}} \theta \frac{1}{k_{n}} \sum_{j=1}^{k_{n}}\left(f_{j}-1\right)+\sqrt{k_{n}} b\left(\log \left(n / k_{n}\right)\right) \frac{1}{k_{n}} \sum_{j=1}^{k_{n}}\left(\frac{\log (n / j)}{\log \left(n / k_{n}\right)}\right)^{\rho}\left(f_{j}-1\right) \\
& +o_{\mathbb{P}}\left(\sqrt{k_{n}} b\left(\log \left(n / k_{n}\right)\right)\right) .
\end{aligned}
$$

Now, an application of Tchebychev's inequality gives that

$$
\frac{1}{k_{n}} \sum_{j=1}^{k_{n}}\left(\frac{\log (n / j)}{\log \left(n / k_{n}\right)}\right)^{\rho}\left(f_{j}-1\right)=o_{\mathbb{P}}(1) .
$$

Then, under our assumptions, Theorem 2 follows by an application of the Central Limit Theorem.

\subsection{Proof of Theorem 3}

From Corollary 1, we have

$$
\begin{aligned}
& \frac{\sqrt{k_{n}}}{\log \left(n / k_{n}\right)}\left(\widehat{\theta}_{n}\left(k_{n}\right)-\theta\right) \\
& =\frac{\sqrt{k_{n}}}{\log \left(n / k_{n}\right)} \frac{1}{k_{n}} \sum_{j=1}^{k_{n}}\left(\theta+b\left(\log \left(n / k_{n}\right)\right) x_{j}\right)\left(1-\frac{x_{j}-\bar{x}_{k_{n}}}{\mu_{2}} \bar{x}_{k_{n}}\right)\left(f_{j}-1\right) \\
& +o_{\mathbb{P}}\left(\frac{\sqrt{k_{n}}}{\log \left(n / k_{n}\right)} b\left(\log \left(n / k_{n}\right)\right)\right) .
\end{aligned}
$$

Since we have (8) and (9), the $o_{\mathbb{P}}$-term is negligible. The first term can be viewed as a sum of a weighted mean of independent and identically distributed variables. Now, using Lyapounov's theorem, we only have to show that

$$
\lim _{k_{n} \rightarrow \infty} \frac{1}{s_{k_{n}}^{4}} \sum_{j=1}^{k_{n}} \mathbb{E} X_{j}^{4}=0,
$$


where $X_{j}=\left(\theta+b\left(\log \left(n / k_{n}\right)\right) x_{j}\right)\left(1-\frac{x_{j}-\bar{x}_{k_{n}}}{\mu_{2}} \bar{x}_{k_{n}}\right)\left(f_{j}-1\right), j=1, \ldots, k_{n}$ and $s_{k_{n}}^{2}=\sum_{j=1}^{k_{n}} \operatorname{Var} X_{j}$. We remark that

$$
s_{k_{n}}^{2} \sim \theta^{2} \sum_{j=1}^{k_{n}}\left(1-\frac{x_{j}-\bar{x}_{k_{n}}}{\mu_{2}} \bar{x}_{k_{n}}\right)^{2} \quad \text { as } n \rightarrow \infty
$$

and

$$
\sum_{j=1}^{k_{n}} \mathbb{E} X_{j}^{4} \sim 9 \theta^{4} \sum_{j=1}^{k_{n}}\left(1-\frac{x_{j}-\bar{x}_{k_{n}}}{\mu_{2}} \bar{x}_{k_{n}}\right)^{4} \quad \text { as } n \rightarrow \infty
$$

from which we deduce by direct computations that

$$
\begin{aligned}
\frac{1}{s_{k_{n}}^{4}} \sum_{j=1}^{k_{n}} \mathbb{E} X_{j}^{4} & \sim \frac{9}{k_{n}} \frac{\mu_{2}^{4}+6\left(\bar{x}_{k_{n}}\right)^{2} \mu_{2}^{3}-4\left(\bar{x}_{k_{n}}\right)^{3} \mu_{2} \mu_{3}+\left(\bar{x}_{k_{n}}\right)^{4} \mu_{4}}{\left[\mu_{2}^{2}+\left(\bar{x}_{k_{n}}\right)^{2} \mu_{2}\right]^{2}} \\
& \sim \frac{9 C_{4}}{k_{n}}
\end{aligned}
$$

by Lemma 5 , Our Theorem 3 now follows from the fact that

$$
s_{k_{n}}^{2} \sim \theta^{2} k_{n} \log ^{2}\left(n / k_{n}\right) \text {. }
$$

\section{Appendix}

In this appendix, we briefly show how to adapt Hall's class of distribution function [16] to the framework of Weibull tail-distributions. We introduce the class of distributions $\mathcal{D}(\alpha, \beta)$ with distribution function given by

$$
1-F(x)=\exp (-H(x)) \text { where } H^{-1}(x):=x^{1 / \alpha}\left(1+x^{-\beta}\right),
$$

$\alpha$ and $\beta$ being two parameters such that

$$
0<\alpha, 0<\beta<1 \text { and } \alpha \beta \leq 1 \text {. }
$$

It is easily seen that under (11), the above class of distributions fulfill assumptions (1) with $\left(R_{\ell}(b, \rho)\right)$ and (4) where $\theta=1 / \alpha, \rho=-\beta, \ell(x)=$ $1+x^{-\beta}$ and $b(x)=-\beta x^{-\beta}$. It is thus possible to obtain distributions with arbitrary $\theta>0$ and $-1<\rho<0$. These results are summarized in Table1, 


\section{Acknowledgement}

The authors are very grateful to the referees for a careful reading of the paper that led so significant improvements of the earlier draft.

\section{References}

[1] Abramowitz, M., Stegun, I., (1972), Handbook of Mathematical Functions, Dover.

[2] Beirlant, J., Teugels, J. and Vynckier, P., (1996) Practical analysis of extreme values, Leuven university press.

[3] Beirlant, J., Bouquiaux, C., Werker, B., (2005), Semiparametric lower bounds for tail index estimation, Journal of Statistical Planning and Inference, to appear.

[4] Beirlant, J., Broniatowski, M., Teugels, J.L., Vynckier, P., (1995), The mean residual life function at great age: Applications to tail estimation, Journal of Statistical Planning and Inference, 45, 21-48.

[5] Beirlant, J., Dierckx, G., Goegebeur, Y., Matthys, G., (1999), Tail index estimation and an exponential regression model, Extremes, $2,177-200$.

[6] Beirlant, J., Dierckx, G., Guillou, A., Starica, C., (2002), On exponential representations of log-spacings of extreme order statistics, Extremes, 5 (2), 157-180.

[7] Broniatowski, M., (1993), On the estimation of the Weibull tail coefficient, Journal of Statistical Planning and Inference, 35, 349366.

[8] Davison, A.C. and Smith, R.L., (1990) Models for exceedances over high thresholds, Journal of the Royal Statistical Society B, 52(3), 393-442.

[9] Diebolt, J., El-Aroui, M., Garrido, M. and Girard, S. (2005) Quasiconjugate Bayes estimates for GPD parameters and application to heavy tails modelling, Extremes, 8, 57-78. 
[10] Diebolt, J., Gardes, L., Girard, S. and Guillou, A., (2008) Bias-reduced extreme quantile estimators of Weibull taildistributions, Statistical Journal of Planning and Inference, 138, 1389-1401.

[11] Feuerverger, A., Hall, P., (1999), Estimating a Tail Exponent by Modelling Departure from a Pareto Distribution, Annals of Statistics, 27, 760-781.

[12] Gardes, L., Girard, S., (2005), Estimating extreme quantiles of Weibull tail-distributions, Communication in Statistics - Theory and Methods, 34, 1065-1080.

[13] Gardes, L. and Girard, S. (2006), Comparison of Weibull tailcoefficient estimators, REVSTAT - Statistical Journal, 4(2), 163188.

[14] Geluk, J.L., de Haan, L., (1987), Regular Variation, Extensions and Tauberian Theorems. Math Centre Tracts, 40, Centre for Mathematics and Computer Science, Amsterdam.

[15] Girard, S., (2004), A Hill type estimate of the Weibull tailcoefficient, Communication in Statistics - Theory and Methods, 33(2), 205-234.

[16] Hall, P. and Welsh, A.H., (1985) Adaptive estimates of parameters of regular variation, Annals of Statistics, 13, 331-341.

[17] Hill, B.M., (1975), A simple general approach to inference about the tail of a distribution, Annals of Statistics, 3, 1163-1174.

[18] Hosking, J., Wallis, J. and Wood, E., (1985) Estimation of the Generalized extreme-value distribution by the method of probability-weighted moments, Technometrics, 27, 251-257.

[19] Matthys, G. and Beirlant, J., (2003) Estimating the extreme value index and high quantiles with exponential regression models, Statistica Sinica, 13(3), 853-880.

[20] Matthys, G., Delafosse, E., Guillou, A. and Beirlant, J., (2004) Estimating catastrophic quantile levels for heavy-tailed distributions, Insurance: Mathematics and Economics, 34(3), 517-537. 
[21] Shorack, G.R., Wellner, J.A., (1986), Empirical Processes with Applications to Statistics, Wiley New York. 


\begin{tabular}{|c|c|c|c|}
\hline Distribution & $\theta$ & $b(x)$ & $\rho$ \\
\hline Absolute Gaussian $|\mathcal{N}|\left(\mu, \sigma^{2}\right)$ & $1 / 2$ & $\frac{1}{4} \frac{\log x}{x}$ & -1 \\
Gamma $\Gamma(\alpha \neq 1, \beta)$ & 1 & $(1-\alpha) \frac{\log x}{x}$ & -1 \\
Weibull $\mathcal{W}(\alpha, \lambda)$ & $1 / \alpha$ & 0 & $-\infty$ \\
$\mathcal{D}(\alpha, \beta)$ & $1 / \alpha$ & $-\beta x^{-\beta}$ & $-\beta$ \\
\hline
\end{tabular}

Table 1: Parameters $\theta, \rho$ and the function $b(x)$ associated to some distributions

\begin{tabular}{|c|c|c|c|c|c|c|c|c|}
\hline Distribution & $\theta$ & $\rho$ & $\mu\left(\widehat{k_{n}}\right)$ & $\sigma\left(\widehat{k_{n}}\right)$ & $\mu\left(\check{\theta}_{n}\right)$ & $\sigma\left(\check{\theta}_{n}\right)$ & $R_{n}$ & $k_{n}^{\text {opt }}$ \\
\hline$\Gamma(0.25,1)$ & 1 & -1 & 105.5 & 62.2 & 1.667 & 0.294 & 1.26 & 186 \\
$\Gamma(4,1)$ & 1 & -1 & 222.7 & 82.1 & 0.548 & 0.051 & 1.13 & 184 \\
$|\mathcal{N}|(0,1)$ & 0.5 & -1 & 246.6 & 81.1 & 0.679 & 0.109 & 1.21 & 189 \\
$\mathcal{W}(0.25,0.25)$ & 4 & $-\infty$ & 305.8 & 59.0 & 4.016 & 0.265 & 1.62 & 350 \\
$\mathcal{W}(4,4)$ & 0.25 & $-\infty$ & 310.4 & 50.9 & 0.249 & 0.013 & 1.43 & 350 \\
$\mathcal{D}(1,0.5)$ & 1 & -0.5 & 281.5 & 71.1 & 0.789 & 0.053 & 1.14 & 43 \\
& & & & & & & & \\
\hline
\end{tabular}

Table 2: Simulation results of the adaptive selection procedure 


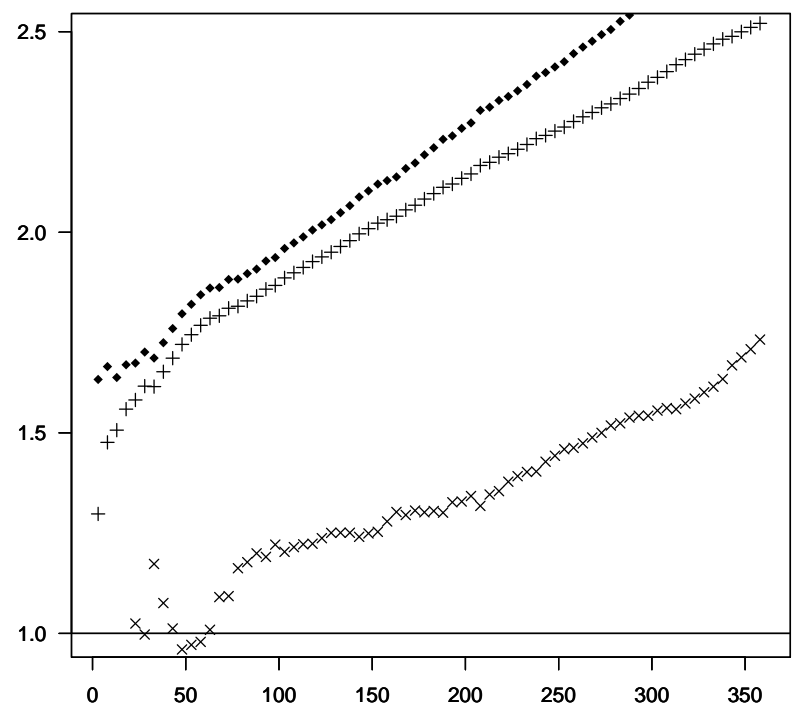

(a) Mean as a function of $k_{n}$

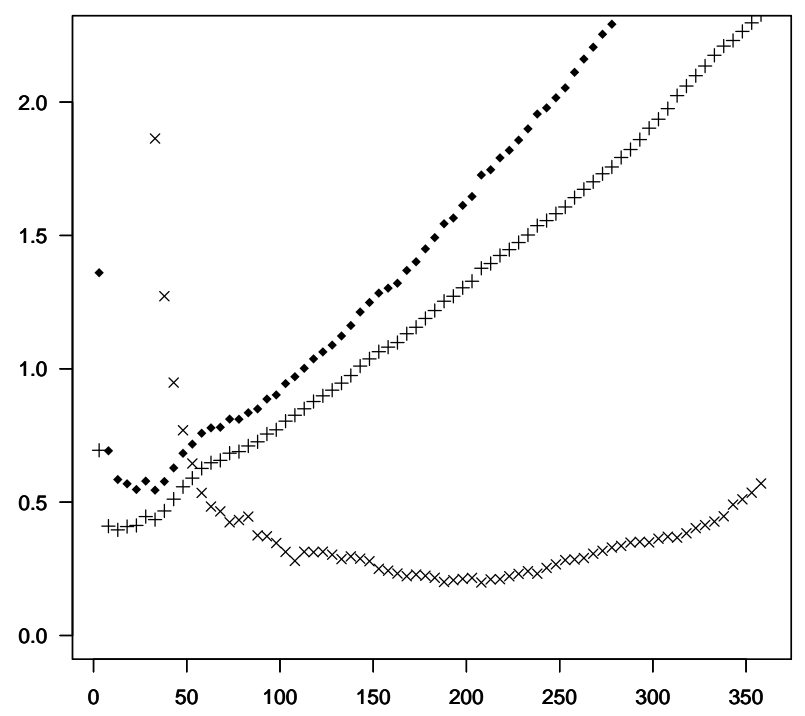

(b) Mean squared error as a function of $k_{n}$

Figure 1: Comparison of estimates $\widehat{\theta}_{n}^{2} 2(\times \times \times), \widetilde{\theta}_{n}(\diamond \diamond \diamond)$ and $\check{\theta}_{n}(+++)$ for the $\Gamma(0.25,1)$ distribution. In (a), the straight line is the true value of $\theta$. 


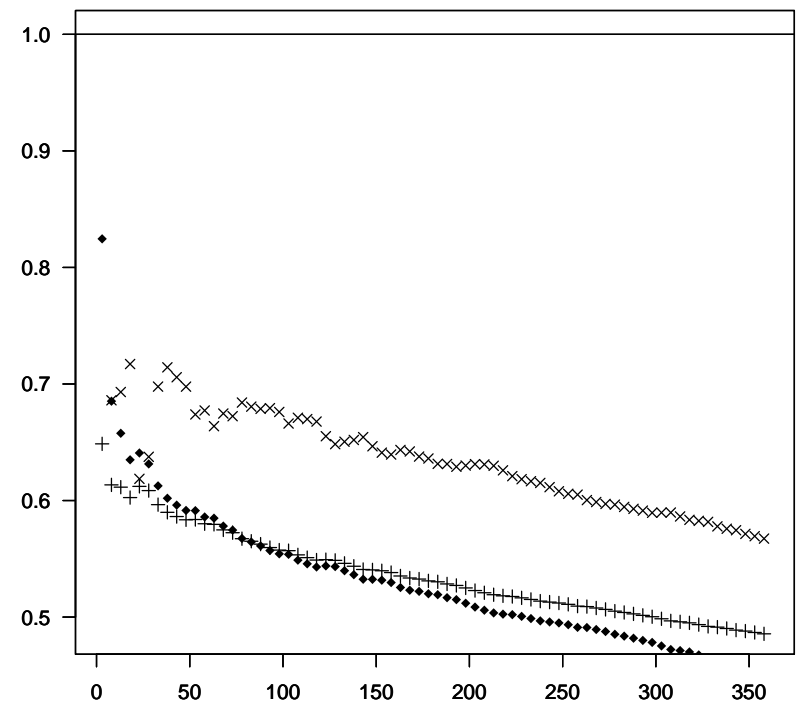

(a) Mean as a function of $k_{n}$

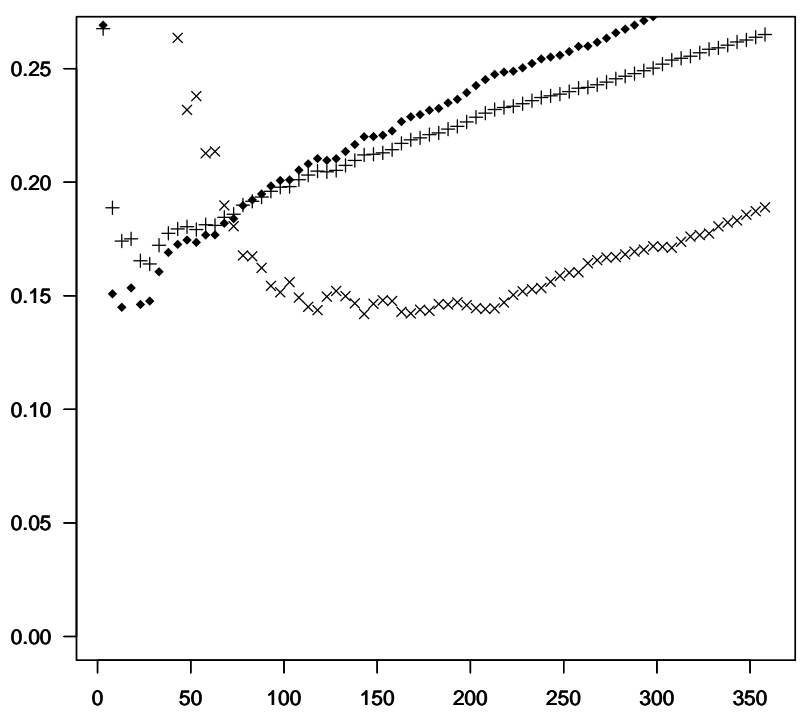

(b) Mean squared error as a function of $k_{n}$

Figure 2: Comparison of estimates $\widehat{\theta}_{n}^{3} 3(\times \times \times), \widetilde{\theta}_{n}(\diamond \diamond \diamond)$ and $\check{\theta}_{n}(+++)$ for the $\Gamma(4,1)$ distribution. In (a), the straight line is the true value of $\theta$. 


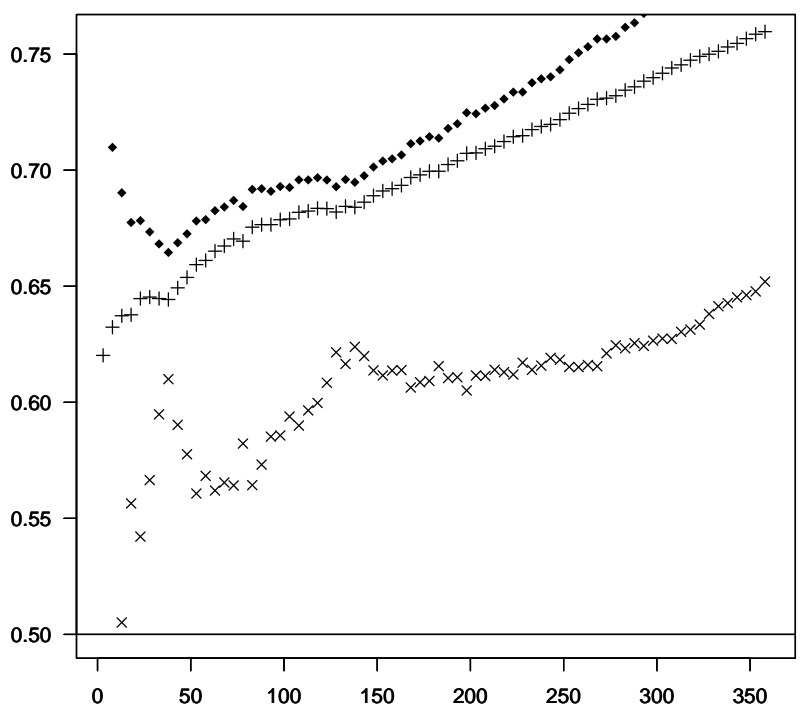

(a) Mean as a function of $k_{n}$

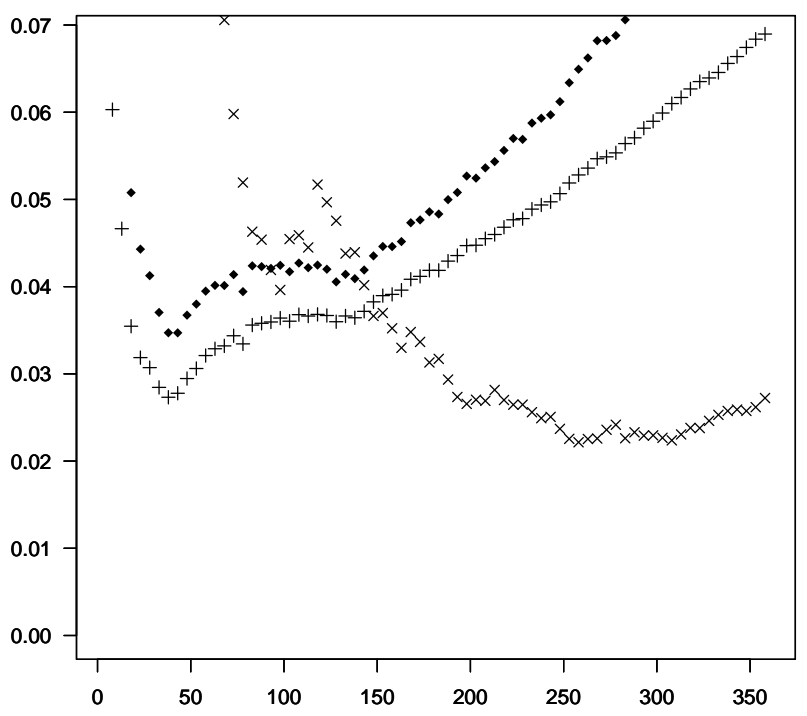

(b) Mean squared error as a function of $k_{n}$

Figure 3: Comparison of estimates $\widehat{\theta}_{n} 4(\times \times \times), \widetilde{\theta}_{n}(\diamond \diamond \diamond)$ and $\check{\theta}_{n}(+++)$ for the $|\mathcal{N}|(0,1)$ distribution. In (a), the straight line is the true value of $\theta$. 


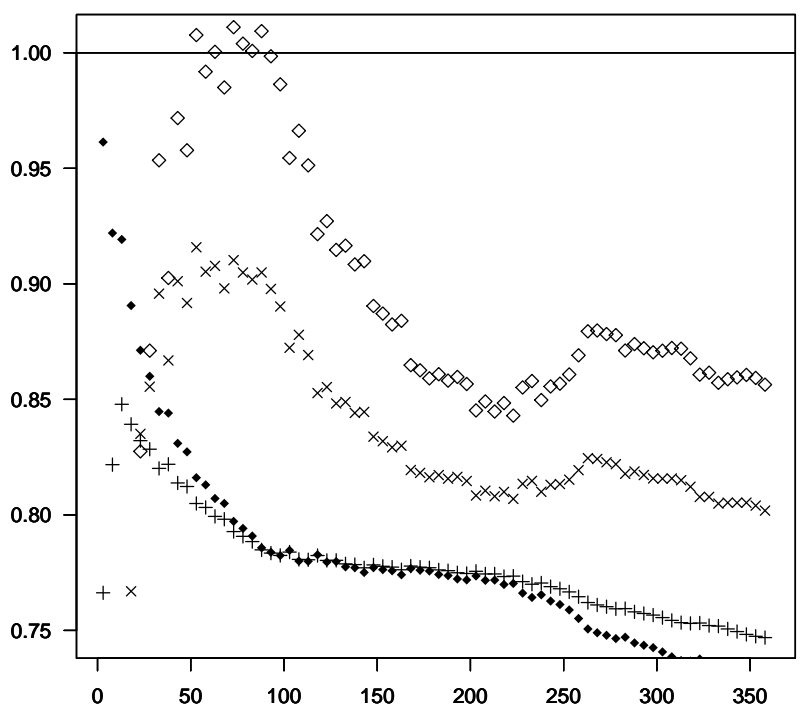

(a) Mean as a function of $k_{n}$

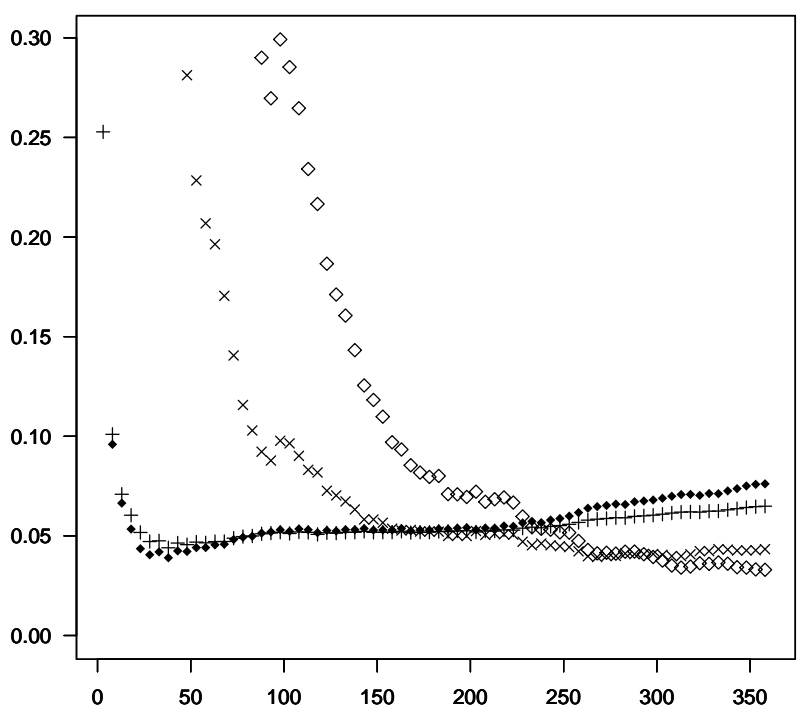

(b) Mean squared error as a function of $k_{n}$

Figure 4: Comparison of estimates $\widehat{2}$ (with the canonical choice $\rho=-1$ : $\times \times \times), \widehat{\theta}_{n}$ (with the true $\left.\rho=-1 / 2: \diamond \diamond \diamond\right), \widetilde{\theta}_{n}(\diamond \diamond \diamond)$ and $\check{\theta}_{n}(+++)$ for the $\mathcal{D}(1,0.5)$ distribution. In (a), the straight line is the true value of $\theta$. 


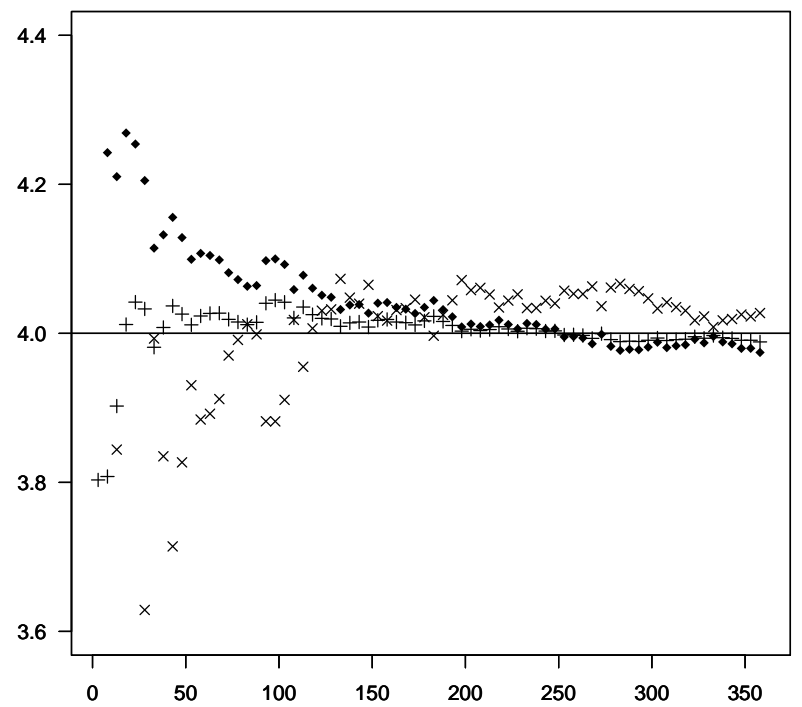

(a) Mean as a function of $k_{n}$

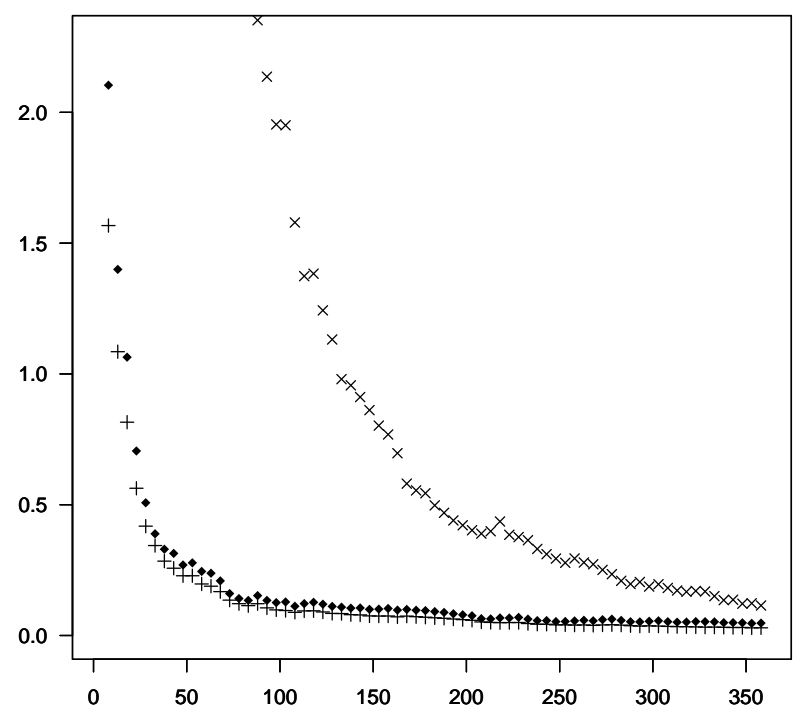

(b) Mean squared error as a function of $k_{n}$

Figure 5: Comparison of estimates $\widehat{\theta}_{n}^{2} 6(\times \times \times), \widetilde{\theta}_{n}(\diamond \diamond \diamond)$ and $\check{\theta}_{n}(+++)$ for the $\mathcal{W}(0.25,0.25)$ distribution. In (a), the straight line is the true value of $\theta$. 


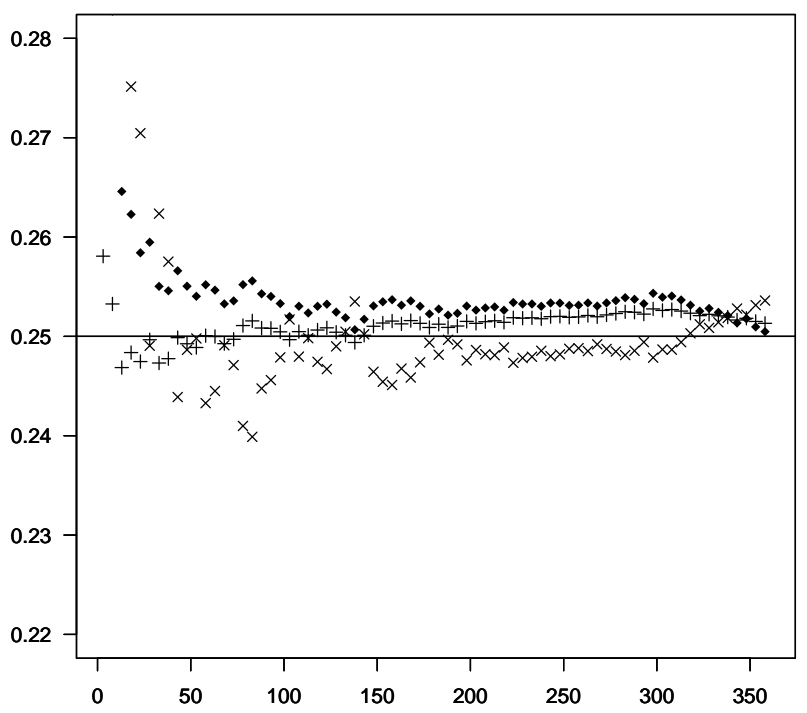

(a) Mean as a function of $k_{n}$

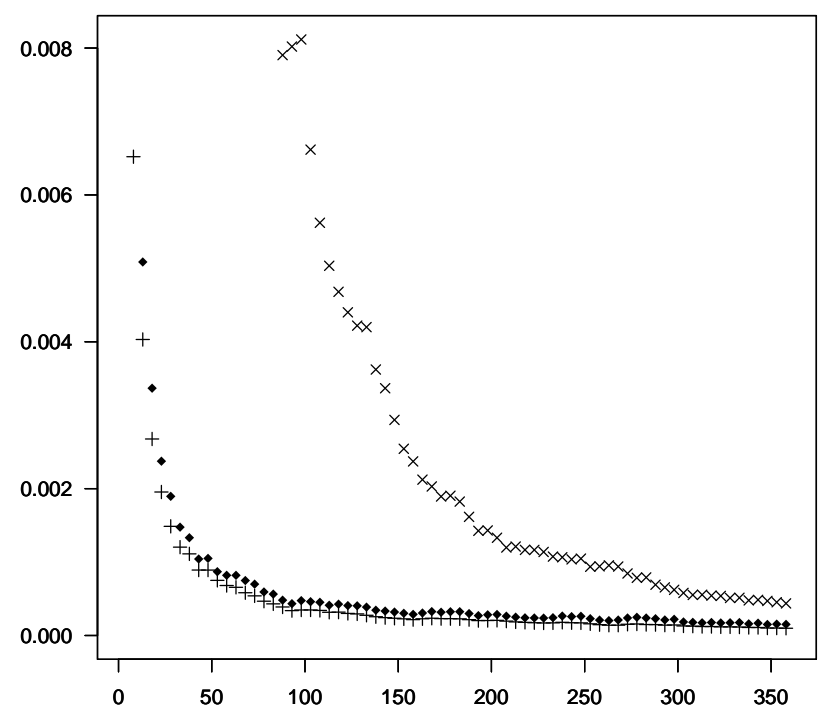

(b) Mean squared error as a function of $k_{n}$

Figure 6: Comparison of estimates $\widehat{\theta}_{n}^{2} 7(\times \times \times), \widetilde{\theta}_{n}(\diamond \diamond \diamond)$ and $\check{\theta}_{n}(+++)$ for the $\mathcal{W}(4,4)$ distribution. In (a), the straight line is the true value of $\theta$. 


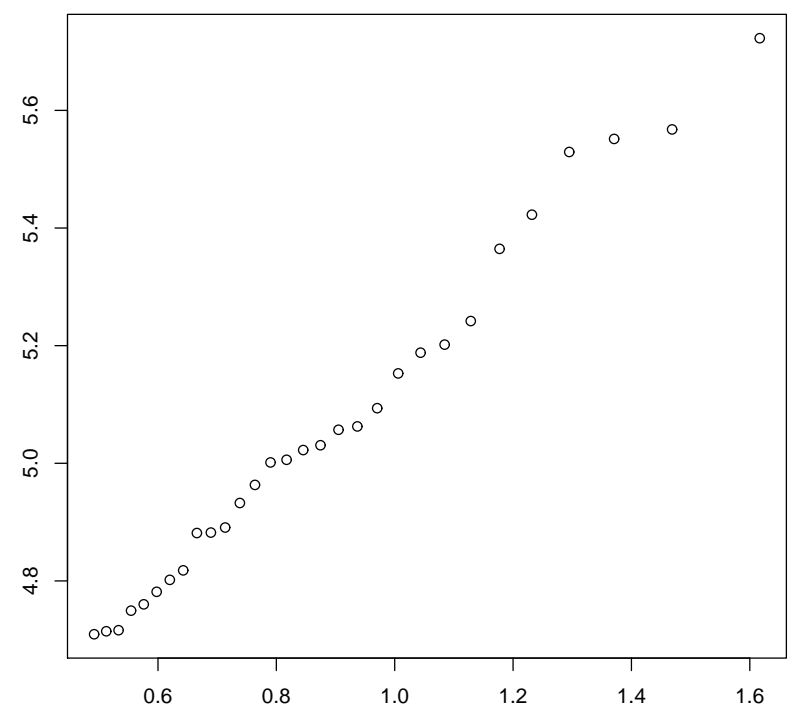

Figure 7: Quantile-quantile plot obtained with $\widehat{k}_{n}=29$ on the Nidd river data. 